\title{
A!
}

This is an electronic reprint of the original article.

This reprint may differ from the original in pagination and typographic detail.

Singh, Shilpi; Mannila, Elsa T.; Golubev, Dmitry S.; Peltonen, Joonas T.; Pekola, Jukka P.

Determining the parameters of a random telegraph signal by digital low pass filtering

Published in:

Applied Physics Letters

DOI:

$10.1063 / 1.5033560$

Published: 11/06/2018

Document Version

Publisher's PDF, also known as Version of record

Please cite the original version:

Singh, S., Mannila, E. T., Golubev, D. S., Peltonen, J. T., \& Pekola, J. P. (2018). Determining the parameters of a random telegraph signal by digital low pass filtering. Applied Physics Letters, 112(24), 1-5. [243101].

https://doi.org/10.1063/1.5033560

This material is protected by copyright and other intellectual property rights, and duplication or sale of all or part of any of the repository collections is not permitted, except that material may be duplicated by you for your research use or educational purposes in electronic or print form. You must obtain permission for any other use. Electronic or print copies may not be offered, whether for sale or otherwise to anyone who is not an authorised user. 


\section{Determining the parameters of a random telegraph signal by digital low pass filtering}

Shilpi Singh, Elsa T. Mannila, Dmitry S. Golubev, Joonas T. Peltonen, and Jukka P. Pekola

Citation: Appl. Phys. Lett. 112, 243101 (2018); doi: 10.1063/1.5033560

View online: https://doi.org/10.1063/1.5033560

View Table of Contents: http://aip.scitation.org/toc/apl/112/24

Published by the American Institute of Physics

\section{Articles you may be interested in}

Dual-mode resonant infrared detector based on film bulk acoustic resonator toward ultra-high sensitivity and antiinterference capability

Applied Physics Letters 112, 243501 (2018); 10.1063/1.5032236

Demonstration of long wavelength infrared type-II InAs/InAs $1-x b_{x}$ superlattices photodiodes on GaSb substrate grown by metalorganic chemical vapor deposition

Applied Physics Letters 112, 241103 (2018); 10.1063/1.5035308

Coordinate transformation resonators

Applied Physics Letters 112, 234101 (2018); 10.1063/1.5027017

Non-volatile and volatile memory behaviour in oxygenated amorphous carbon electrochemical metallisation devices

Applied Physics Letters 112, 242903 (2018); 10.1063/1.5029402

Local carrier distribution imaging on few-layer $\mathrm{MoS}_{2}$ exfoliated on $\mathrm{SiO}_{2}$ by scanning nonlinear dielectric microscopy

Applied Physics Letters 112, 243102 (2018); 10.1063/1.5032277

Tunneling anisotropic magnetoresistance in fully epitaxial magnetic tunnel junctions with different barriers Applied Physics Letters 112, 242404 (2018); 10.1063/1.5027909

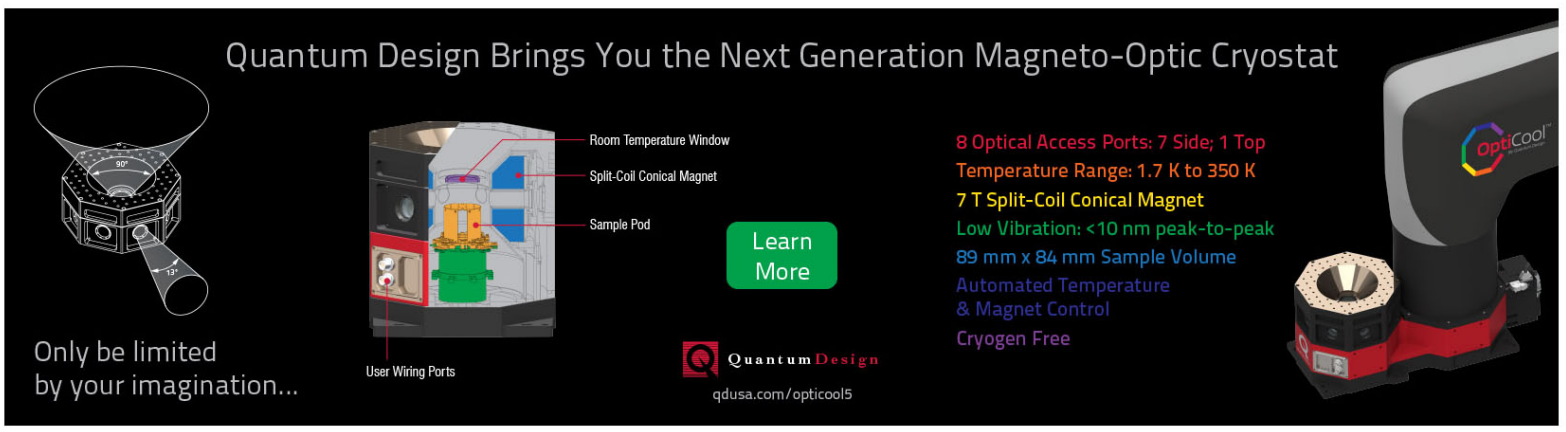




\title{
Determining the parameters of a random telegraph signal by digital low pass filtering
}

\author{
Shilpi Singh, Elsa T. Mannila, Dmitry S. Golubev, Joonas T. Peltonen, and Jukka P. Pekola \\ QTF Centre of Excellence, Department of Applied Physics, Aalto University, FI-00076 Aalto, Finland
}

(Received 6 April 2018; accepted 26 May 2018; published online 11 June 2018)

\begin{abstract}
We propose a method to determine the switching rates of a random telegraph signal. We apply digital low pass filtering with varying bandwidths to the raw signal, evaluate the cumulants of the resulting distributions, and compare them with the analytical prediction. This technique is useful in the case of a slow detector with response time comparable to the time interval between the switching events. We demonstrate the efficiency of this method by analyzing random telegraph signals generated by individual charge tunneling events in metallic single-electron transistors. Published by AIP Publishing. https://doi.org/10.1063/1.5033560
\end{abstract}

Telegraph noise, or random switching of the electric current between two levels, is often observed in electronic devices. ${ }^{1}$ In the context of nanotechnology, it is particularly important for semiconducting ${ }^{2,3}$ and single-electron transistors (SETs), ${ }^{4-6}$ as well as for solid-state quantum bits (qubits). ${ }^{7-9}$ Switching rates between the two current levels and their dependence on temperature, gate voltage, or other parameters provide valuable information about the physical nature of the two-level systems generating the noise. The standard way of finding these rates is based on threshold detection algorithms, in which the detector current values below or above certain threshold are assigned to the first or the second state of the two-level system.

Accurate determination of the switching rates may be hampered by white and $1 / f$-noises present in the output signal of the detector and by the long response time of the latter. Several methods of correcting the errors caused by these effects have been developed in the past. For example, the white noise is efficiently suppressed by digital low pass filtering. Unfortunately, during this procedure, switching events separated by short time intervals are lost as well. ${ }^{10}$ In order to take that into account, Naaman and Aumentado ${ }^{10}$ have introduced two additional states of the system corresponding to the errors occurring when the detector does not immediately switch after the jump in the telegraph signal and introduced the decay rate of these states as an additional parameter. Yuzhelevski et al. ${ }^{11}$ have proposed an iterative procedure of the analysis of noisy data, in which the thresholds for the jumps between the two levels are adjusted based on the values of the switching rates found in the previous iteration. Martin-Martinez et al. ${ }^{12}$ have introduced the weighted time lag method relying on the analysis of correlations between the neighboring points in the digitized noisy signal. Küng et al. ${ }^{13}$ have proposed a cross-correlation technique with two detectors, and Prance et al. have used the wavelet edge detection technique. ${ }^{14}$ All these techniques have been tested in practice and proved to be efficient. ${ }^{15-17}$ Additional complications arise if several two level systems contribute to the noise and the current switches between multiple levels. Awano et al. have developed an algorithm, based on the theory of Markov chains ${ }^{18}$ and Monte Carlo simulations, capable of determining the parameters of all two level systems. ${ }^{19}$ A similar approach has been used by Puglisi and Pavan, who have analyzed noise in random access memories. ${ }^{20}$ Giusi et al. have proposed an algorithm of separation of the two and multilevel telegraph noise from background $1 / f$-noise. ${ }^{21}$

In this letter, we analyse a random telegraph signal which switches between two levels. We propose an approach, which has advantages if the detector is slow and a threshold algorithm would produce too many errors to be corrected by the techniques mentioned above. For that purpose, we use an analytical expression for the statistical distribution of the output current of the detector recording random telegraph noise and having an arbitrary bandwidth. This distribution has been derived by Fitzhugh ${ }^{22}$ and used, for example, in the analysis of switching rates between the charging states of superconducting double dots by Lambert et al. ${ }^{23}$ We propose to reduce the effective bandwidth of the detector below its maximum value, set by hardware, by digital low pass filtering of the output signal. Varying the bandwidth in this way and comparing the cumulants of the resulting distributions with corresponding analytical expressions, one can determine the switching rates. Our method should work even in the limit of a very slow detector, when the two current levels, between which the switching is happening, cannot be reliably determined.

In order to test the theory, we have studied random telegraph noise in a system of two capacitively coupled singleelectron transistors (SETs). ${ }^{25}$ One of them is highly resistive and serves as a source of telegraph noise caused by random switches between the two charge states of the SET island (turquoise in Fig. 1). We denote these states as 0 (no extra electron on the island) and 1 (one extra electron on the island). The second transistor with lower resistance is used as a detector (blue in Fig. 1). We voltage bias the detector $\left(V_{\mathrm{b}, \mathrm{det}}\right)$ above the Coulomb blockade threshold, to have measurable current flowing through it, and tune the gate voltage $\left(V_{\mathrm{g}, \text { det }}\right)$ to the most sensitive point. The detector current $(I)$ monitors the charging states of the noise source SET and switches between the two values $I_{0}$ and $I_{1}$ corresponding to the states 0 and 1 . We denote the rate of the transition $0 \rightarrow 1$ by $\gamma_{\uparrow}$ and the rate of the opposite transition, $1 \rightarrow 0$, by $\gamma_{\downarrow}$. On top of purely telegraph signal, $I_{\mathrm{tel}}(t)$, the measurement 


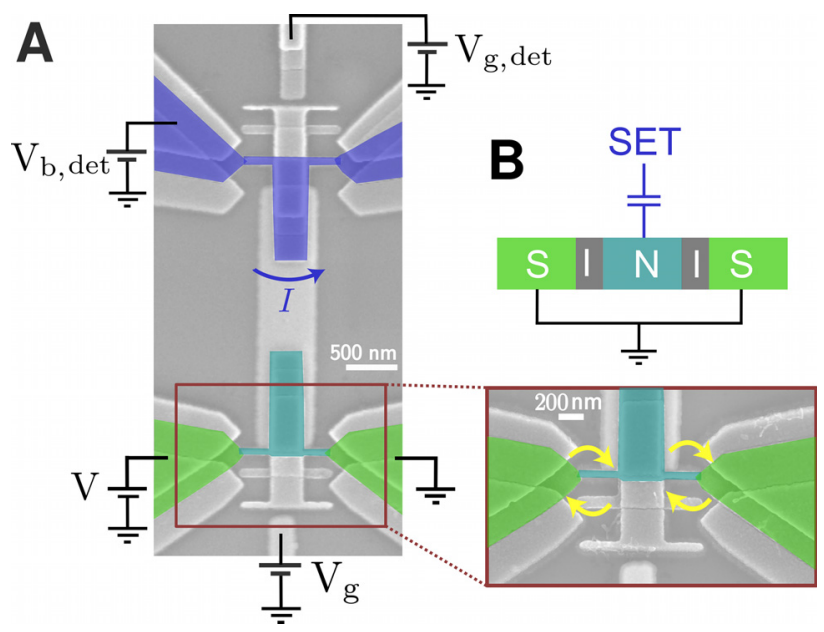

FIG. 1. Experimental setup. (a) Pseudo-colored scanning electron micrograph of the detector SET (blue, top) and another SET used as the noise source. (b) Top: schematic sketch of the measurement setup. Bottom: Zoomed view of the lower SET, which has two superconducting leads (S, green) and normal-metallic island (N, turquoise), separated by an insulating barrier (I). Both devices are fabricated by electron beam lithography and three-angle shadow evaporation. ${ }^{25}$ The gate voltages $\mathrm{V}_{\mathrm{g} \text {,det }}$ and $\mathrm{V}_{\mathrm{g}}$ are used to control the tunneling rates in the detector and source SET, respectively. The source bias voltage $V$ was set to 0 for sample A and was varied to change the tunneling rates in sample $\mathrm{B}$.

setup adds the noise $\xi(t)$. Thus, the output current of the detector SET is

$$
I_{d}(t)=I_{\mathrm{tel}}(t)+\xi(t) .
$$

We have fabricated two samples with similar design (Fig. 1). For sample A, we have recorded $50 \mathrm{~s}$ long time traces of the detector current at zero source SET bias with the sampling rate of $50 \mathrm{kHz}$ (time step $\tau_{0}=20 \mu \mathrm{s}$ ) by digitizing the output of the current preamplifier with an analog-to-digital converter. Part of such a trace is shown in Fig. 2(a). Sample A produces a clear telegraph signal which can be analyzed by standard methods. We use it as a reference to test the predictions of our model. The detector of the second sample (sample B) has been embedded in a radio-frequency resonant
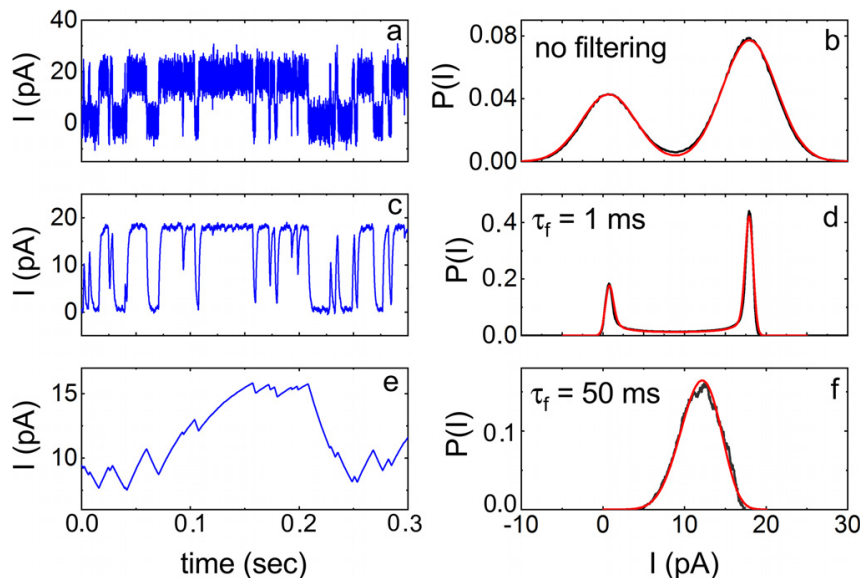

FIG. 2. Sample A: Time dependence of the current in sample A before (a) and after the filtering [(c) and (e)], and corresponding distributions [(b), (d), and (f)]. In (b), (d), and (f), black curves are experimental data, and red curves are the fits with Eqs. (5) and (6). Fit parameters are indicated in the text and caption of Fig. 3 . circuit and instead of the current, we monitor the transmission $\left|s_{21}\right|^{2}$ through the circuit at $588 \mathrm{MHz}$. This sample has also shown a good telegraph signal with zero bias applied to the source SET [Fig. 4(a)]. We have deliberately applied higher bias to it in order to increase the transition rates and to complicate the detection of switching events [Figs. 4(c) and 4(e)]. For this sample, we have recorded $10 \mathrm{~s}$ long traces of the transmission coefficient $\left|s_{21}\right|^{2}$ with a time step of $\tau_{0}=0.1 \mathrm{~ms}$. Under these conditions, a typical histogram has only one peak [Figs. 4(d) and 4(f)], and the threshold algorithm for determining the rates cannot be applied. We will demonstrate that our approach works also in this case.

We will now briefly describe our theoretical model. We assume that the output current of the detector SET (1) goes through a low pass filter with the bandwidth $\tau_{f}^{-1}$, which transforms it as follows:

$$
I(t)=\frac{1}{\tau_{f}} \int_{-\infty}^{t} d t^{\prime} e^{-\left(t-t^{\prime}\right) / \tau_{f}}\left[I_{\mathrm{tel}}\left(t^{\prime}\right)+\xi\left(t^{\prime}\right)\right] .
$$

We have chosen this type of filtering because it emulates an $R C$ low pass filter, which is common in experiments, and because it allows an exact solution of the problem. Our goal is to find the distribution of the filtered current $I(t)$. As a first step, we solve the problem without noise and put $\xi(t)=0$. We introduce two current distributions: one corresponding to state 0 of the source SET, which we denote as $\mathcal{P}_{0}(t, I)$, and the second one, $\mathcal{P}_{1}(t, I)$, corresponding to state 1 . The evolution of these two distributions in time is described by theory of stochastic jump processes (see, for example, Ref. 24 for details). The corresponding evolution equations read

$$
\begin{aligned}
& \partial_{t} \mathcal{P}_{0}+\tau_{f}^{-1} \partial_{I}\left[\left(I_{0}-I\right) \mathcal{P}_{0}\right]=-\gamma_{\uparrow} \mathcal{P}_{0}+\gamma_{\downarrow} \mathcal{P}_{1}, \\
& \partial_{t} \mathcal{P}_{1}+\tau_{f}^{-1} \partial_{I}\left[\left(I_{1}-I\right) \mathcal{P}_{1}\right]=\gamma_{\uparrow} \mathcal{P}_{0}-\gamma_{\downarrow} \mathcal{P}_{1}
\end{aligned}
$$

The stationary solution of these equations can be found analytically. ${ }^{22}$ It reads

$$
\mathcal{P}_{0}(I)=\frac{I_{1}-I}{I_{1}-I_{0}} \mathcal{P}(I), \quad \mathcal{P}_{1}(I)=\frac{I-I_{0}}{I_{1}-I_{0}} \mathcal{P}(I),
$$

where $\mathcal{P}(I)=\mathcal{P}_{0}(I)+\mathcal{P}_{1}(I) \operatorname{reads}^{22}$

$$
\mathcal{P}(I)=\frac{\Gamma\left(\gamma_{\Sigma} \tau_{f}\right)}{\Gamma\left(\gamma_{\uparrow} \tau_{f}\right) \Gamma\left(\gamma_{\downarrow} \tau_{f}\right)} \frac{\left(I-I_{0}\right)^{\gamma_{\uparrow} \tau_{f}-1}\left(I_{1}-I\right)^{\gamma_{\downarrow} \tau_{f}-1}}{\left(I_{1}-I_{0}\right)^{\gamma_{\Sigma} \tau_{f}-1}} .
$$

Here, $\Gamma(x)$ is the gamma function and $\gamma_{\Sigma}=\gamma_{\uparrow}+\gamma_{\downarrow} \cdot \mathcal{P}(I)$ is the distribution of the filtered current (2), which we are looking for. It has the form of a beta distribution, well known in statistics. The distribution of Eq. (5) differs from the one derived for a filter with a sharp cutoff, $I(t)=\frac{1}{\tau_{f}} \int_{t-\tau_{f}}^{t} d t^{\prime}$ $I_{\text {tel }}\left(t^{\prime}\right)$, in Ref. 25, and it does not have a universal form predicted in Ref. 26. However, in the limit $\gamma_{\Sigma} \tau_{f} \gg 1$, all these distributions approach the Gaussian form with the same parameters. In the presence of noise, expression (5) should be convolved with the distribution of the filtered noise $W_{\xi}(I)=\left\langle\delta\left(I-\frac{1}{\tau_{f}} \int_{-\infty}^{t} d t^{\prime} e^{-\left(t-t^{\prime}\right) / \tau_{f}} \xi\left(t^{\prime}\right)\right)\right\rangle$, where $\langle\ldots\rangle$ implies averaging over $\xi(t)$ and takes the form 


$$
P(I)=\int_{I_{0}}^{I_{1}} d I^{\prime} W_{\xi}\left(I-I^{\prime}\right) \mathcal{P}\left(I^{\prime}\right) .
$$

The average current evaluated with the distribution (6) does not depend on time $\tau_{f}$

$$
\langle I\rangle=\left(\gamma_{\downarrow} I_{0}+\gamma_{\uparrow} I_{1}\right) / \gamma_{\Sigma} .
$$

The second cumulant $\mathcal{C}_{2}=\left\langle(I-\langle I\rangle)^{2}\right\rangle$ has the form

$$
\mathcal{C}_{2}\left(\tau_{f}\right)=\frac{\gamma_{\uparrow} \gamma_{\downarrow}\left(I_{1}-I_{0}\right)^{2}}{\gamma_{\Sigma}^{2}\left(1+\gamma_{\Sigma} \tau_{f}\right)}+\sigma_{\xi}^{2}\left(\tau_{f}\right),
$$

where $\sigma_{\xi}^{2}\left(\tau_{f}\right)$ is the variance of the filtered noise. This parameter is expressed via the noise spectral power, $S(\omega)=\int d t e^{i \omega t}$ $\langle\xi(t) \xi(0)\rangle$, and reads

$$
\sigma_{\xi}^{2}\left(\tau_{f}\right)=\int_{\omega_{\min }}^{\infty} \frac{d \omega}{\pi} \frac{S(\omega)}{1+\omega^{2} \tau_{f}^{2}} .
$$

Typically, $\xi(t)$ is the sum of white and $1 / f$-noises, so that $S(\omega)=2 \eta+A /|\omega|$. In this case, one finds

$$
\sigma_{\xi}^{2}\left(\tau_{f}\right)=\left(\sigma_{0}^{2}-\sigma_{\infty}^{2}\right)\left(\tau_{0} / \tau_{f}\right)+\sigma_{\infty}^{2},
$$

where $\sigma_{0}^{2}=\sigma_{\infty}^{2}+\eta / \tau_{0}$ is the variance of the unfiltered current digitized with the time step $\tau_{0}$, and $\sigma_{\infty}^{2}=(A / 2 \pi) \ln [1$ $\left.+\left(\omega_{\min } \tau_{f}\right)^{-2}\right]$ is the contribution of $1 / f$-noise. The latter is almost independent of $\tau_{f}$ until it exceeds $\omega_{\min }^{-1} . \sigma_{\infty}^{2}$ may also include the contribution of noise at $50 \mathrm{~Hz}$ due to pick-up from power lines.

The third and the fourth cumulants of the current, $\mathcal{C}_{3}=\left\langle(I-\langle I\rangle)^{3}\right\rangle$ and $\mathcal{C}_{4}=\left\langle(I-\langle I\rangle)^{4}\right\rangle-3 \mathcal{C}_{2}^{2}$, respectively, are not sensitive to the Gaussian white noise. Normalized to their values at $\tau_{f}=0$, they read

$$
\begin{gathered}
\frac{\mathcal{C}_{3}\left(\tau_{f}\right)}{\mathcal{C}_{3}(0)}=\frac{2}{\left(1+\gamma_{\Sigma} \tau_{f}\right)\left(2+\gamma_{\Sigma} \tau_{f}\right)}+\frac{\mathcal{C}_{3, \infty}}{\mathcal{C}_{3}(0)} \\
\frac{\mathcal{C}_{4}\left(\tau_{f}\right)}{\mathcal{C}_{4}(0)}=\frac{\left(\gamma_{\uparrow}-\gamma_{\downarrow}\right)^{2}\left(1+\gamma_{\Sigma} \tau_{f}\right)-\gamma_{\uparrow} \gamma_{\downarrow}\left(2+\gamma_{\Sigma} \tau_{f}\right)}{\gamma_{\uparrow} \gamma_{\downarrow}\left(1+\gamma_{\Sigma} \tau_{f}\right)^{2}\left(2+\gamma_{\Sigma} \tau_{f}\right)\left(3+\gamma_{\Sigma} \tau_{f}\right)}+\frac{\mathcal{C}_{4, \infty}}{\mathcal{C}_{4}(0)} .
\end{gathered}
$$

The third cumulant of the unfiltered current reads $\mathcal{C}_{3}(0)$ $=\mathcal{C}_{3, \infty}+\gamma_{\uparrow} \gamma_{\downarrow}\left(\gamma_{\downarrow}-\gamma_{\uparrow}\right)\left(I_{1}-I_{0}\right)^{3} / \gamma_{\Sigma}^{3}$. The expression for $\mathcal{C}_{4}(0)$ is rather long, and we skip it for simplicity. The long time limiting values $\mathcal{C}_{3, \infty}$ and $\mathcal{C}_{4, \infty}$ account for the effect of non-Gaussian $1 / f$-noise and weakly depend on $\tau_{f}$. Importantly, the $\tau_{f}$-dependent parts of the normalized cumulants [first terms in r.h.s. of Eqs. (11) and (12)] do not contain the current levels $I_{0}$ and $I_{1}$, which are impossible to determine for a slow detector with a single peak current distribution [see, e.g., Figs. 2(f) and 4(d)]. Equations (11) and (12) provide the basis for finding the rates in this case.

Having developed the theoretical model, we test it with the data recorded from the reference sample A. For this sample, the distribution of the unfiltered current is well described by the sum of the two Gaussian peaks centered around the currents $I_{0}=0.71 \mathrm{pA}$ and $I_{1}=17.97 \mathrm{pA}$ and having the width $\sigma_{0}=3.32 \mathrm{pA}$ [Fig. 2(b)].
Next, we numerically generate filtered current time traces (2) for different values of $\tau_{f}$. Technically, we first perform discrete Fourier transformation of the unfiltered current generating a sequence of Fourier components $\tilde{I}_{k}$ $=\sum_{j} I_{j} e^{-2 \pi i(i-1)(k-1) / N} / \sqrt{N}$, where $N$ is the total number of points $I_{j}$ in the dataset. Afterwards, we multiply them by the filtering function

$$
F_{k}=\frac{1-e^{-\tau_{0} / \tau_{f}}}{1-e^{-\tau_{0} / \tau_{f}} e^{-2 \pi i(k-1) / N}},
$$

which is the discrete Fourier transform of the exponent appearing in Eq. (2). Finally, we apply the inverse Fourier transformation. Two filtered time traces generated in this way are shown in Figs. 2(c) and 2(e) and one more for sample B in Fig. 4(e). We have generated a series of current distributions with different filtering times and fitted them with Eqs. (5) and (6). We have achieved the best fit of the data with the switching rates $\gamma_{\uparrow}=180 \mathrm{~Hz}$ and $\gamma_{\downarrow}=100 \mathrm{~Hz}$ and assuming Gaussian form of the noise current distribution $W(I)=\exp \left(-I^{2} / 2 \sigma^{2}\right) / \sqrt{2 \pi} \sigma$, where $\sigma$ is defined by Eq. (10) with $\sigma_{\infty}=1.04 \mathrm{pA}$. With these parameters, we have fitted current histograms in the wide range of averaging times $0<\tau_{f}<0.5$ s. Examples of such fits are shown in Figs. 2(d), 2(f), and 3(b).

For comparison, we have also determined the switching rates in a usual way. For that purpose, we have plotted the distributions $h_{0}(t)$ and $h_{1}(t)$ of the lifetimes of the states 0 and 1 , respectively. The lifetimes have been obtained from the current trace filtered with $\tau_{f}=1 \mathrm{~ms}$, for which we have applied a standard threshold detection algorithm with the threshold between the states 0 and 1 placed at $\left(I_{0}+I_{1}\right) / 2$. In Fig. 3(a), we have compared the resulting distributions with the formula derived in Ref. 10

$$
h_{0,1}=\frac{2 \gamma_{\uparrow, \downarrow} \gamma_{\mathrm{det}} e^{-\lambda t / 2} \sinh \left(\sqrt{\lambda^{2}-4 \gamma_{\uparrow, \downarrow} \gamma_{\mathrm{det}}} t / 2\right)}{\sqrt{\lambda^{2}-4 \gamma_{\uparrow, \nu} \gamma_{\mathrm{det}}}} .
$$

Here, $\lambda=\gamma_{\Sigma}+\gamma_{\text {det }}$ and $\gamma_{\text {det }}=\left(\tau_{f} \ln 2\right)^{-1}=1443 \mathrm{~Hz}$ is the effective detector bandwidth. We have found very good agreement between theory and experiment with the same values of the switching rates as before, see Fig. 3(a).
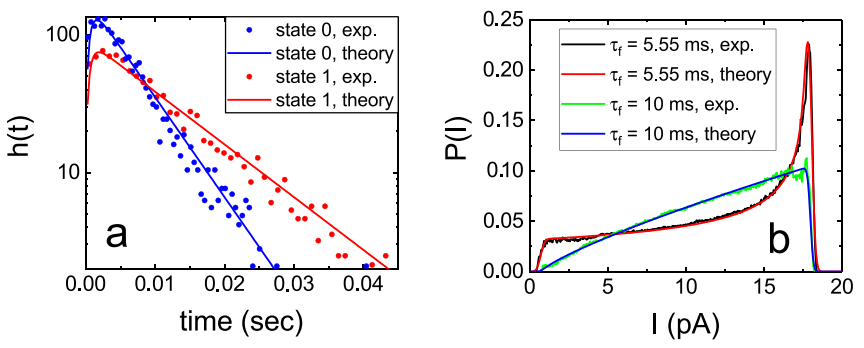

FIG. 3. Calculation of rates. (a) Distributions of the life-times of the states 0 and 1 of sample A extracted from the current time trace filtered with $\tau_{f}=1$ ms (symbols) based on conventional threshold detection. Solid lines show Eq. (14) with the rates $\gamma_{\uparrow}=180 \mathrm{~Hz}$ and $\gamma_{\downarrow}=100 \mathrm{~Hz}$. (b) Distributions of the detector current at $\tau_{f}=\gamma_{\uparrow}^{-1}=1 / 180 \mathrm{~s}$ and $\tau_{f}=\gamma_{\downarrow}^{-1}=1 / 100 \mathrm{~s}$. Fits are based on Eqs. (15) and (6). 

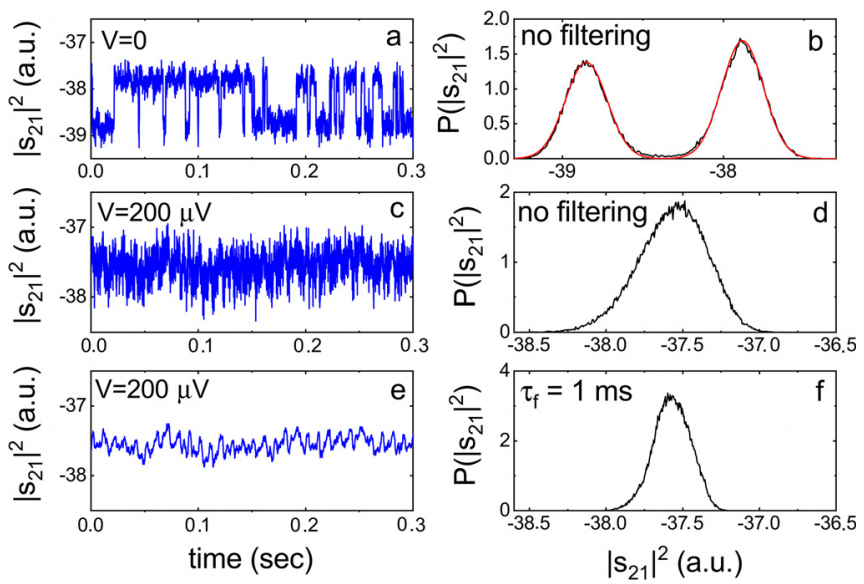

FIG. 4. Sample B: Time traces of $\left|s_{21}\right|^{2}$ at zero bias and dimensionless gate voltage (here, $C_{g}$ is the gate capacitance) $C_{g} V_{g} / e=0.48$, which is close to the maximum of the conductance peak, with the rates $\gamma_{\uparrow}=220 \mathrm{~Hz}, \gamma_{\downarrow}$ $=180 \mathrm{~Hz}$ (a); and at $V=200 \mu \mathrm{V}, C_{g} V_{g} / e=0.42$ [(c) and (e)]. No filtering was applied to the traces (a) and (c), while the trace (e) has been filtered with $\tau_{f}=1 \mathrm{~ms}$. The corresponding distributions are shown in (b), (d), and (f). In (d) and (f), theory fits are absent because the values of the transmission coefficient, between which the switching is happening, are not precisely known at $V=200 \mu \mathrm{V}$. Red curve in (b) is a fit with two Gaussian peaks.

The shape of the current distribution changes with the filtering time $\tau_{f}$ in a way illustrated in Fig. 2. At short $\tau_{f}$, the white noise is suppressed and the two peaks in the distribution become sharper. With growing $\tau_{f}$, the two peaks disappear one after another exactly at times $\tau_{f}=\gamma_{\uparrow}^{-1}$ and $\tau_{f}=\gamma_{\downarrow}^{-1}$. At $\tau_{f}=\gamma_{\uparrow}^{-1}$, the current distribution (5) takes a particularly simple form

$$
\mathcal{P}\left(\tau_{f}=\gamma_{\uparrow}^{-1}, I\right)=\frac{\gamma_{\downarrow}\left(I_{1}-I\right)^{\gamma_{\downarrow} / \gamma_{\uparrow}-1}}{\gamma_{\uparrow}\left(I_{1}-I_{0}\right)^{\gamma_{\downarrow} / \gamma_{\uparrow}}}
$$

shown in Fig. 3(b). The distribution at $\tau_{f}=\gamma_{\downarrow}^{-1}$ is given by the same formula with the interchanged rates $\gamma_{\uparrow} \leftrightarrow \gamma_{\downarrow}$.

Next, we apply our model to sample $\mathrm{B}$, replacing the current by the transmission coefficient, $I \rightarrow\left|s_{21}\right|^{2}$. The distribution of the latter has a form of a single skewed peak, which does not split into two peaks even after filtering. The shape of the peak resembles the distribution of the heavily filtered current of sample A, cf. Figs. 2(f) and 4(d). Obviously, standard threshold detection of the switching events is not possible in this case. In order to estimate the switching rates, we evaluate the cumulants $\mathcal{C}_{3}$ and $\mathcal{C}_{4}$, plot them as functions of the time $\tau_{f}$ (Fig. 5), and fit the result with Eqs. (11) and (12). The data from sample A (blue) can be reasonably well fitted with the same parameters as before, which once again confirms the validity of our model. Fitting the third cumulant for sample $B$ (red), we have determined the total switching rate in this sample, $\gamma_{\Sigma}=9 \pm 2 \mathrm{kHz}$. Next, fitting the fourth cumulant, we determine the two rates separately, $\gamma_{\uparrow}=7 \mathrm{kHz}$ and $\gamma_{\downarrow}=2 \mathrm{kHz}$. Strong low frequency noise and finite value of the time step limit the accuracy of the result, especially for the fourth cumulant. We have verified that these values of the rates are consistent with the ones obtained by extrapolation from the lower bias regime, where the standard threshold-based methods are still applicable, see Fig. 6. To account for the finite sampling rate in the simplest approximation, we have replaced $\tau_{f} \rightarrow \tau_{f}^{\text {eff }}=\tau_{0} /\left(e^{\tau_{0} / \tau_{f}}-1\right)$ in
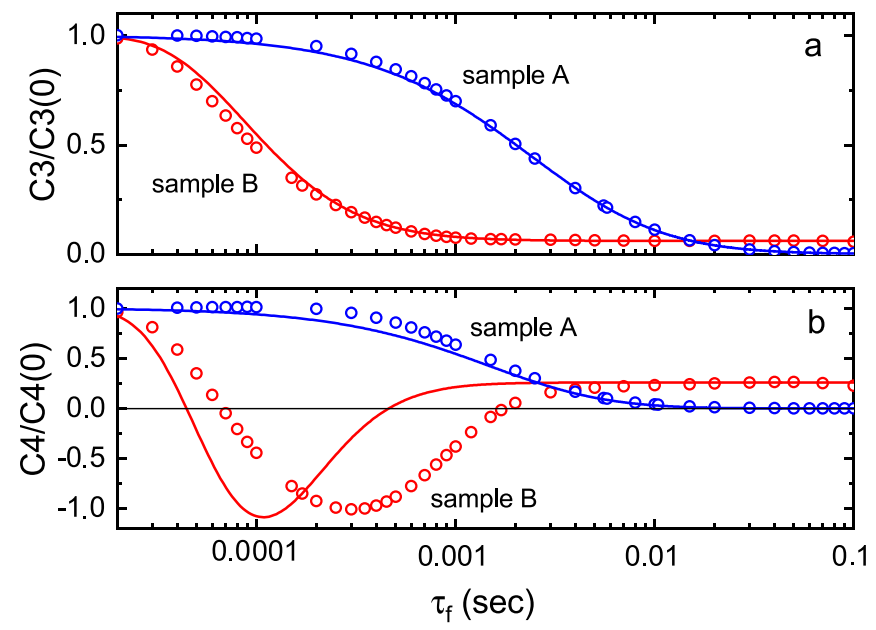

FIG. 5. Using cumulants as rate calculator. Normalized third (a) and fourth (b) cumulants of the distributions of the current for sample A (blue) and of the transmission coefficient for sample B (red). Symbols are experimental data, solid lines theory fits with Eqs. (11) and (12). For sample A, we used the parameters reported in the text and $\mathcal{C}_{3, \infty}=\mathcal{C}_{4, \infty}=0$. For sample $\mathrm{B}$, we have found $\mathcal{C}_{3, \infty}=0.06 \mathcal{C}_{3}(0)$ and $\mathcal{C}_{4, \infty}=0.24 \mathcal{C}_{4}(0)$. For both samples, the third cumulants are negative, $\mathcal{C}_{3}\left(\tau_{f}\right)<0$, implying $\gamma_{\uparrow}>\gamma_{\downarrow}$.

Eqs. (11) and (12). This replacement is justified by a lowfrequency expansion of the function (13) and by fitting it to the form $1 /\left(1-i \omega \tau_{f}^{\text {eff }}\right)$, corresponding to exponential decay in the time domain.

In conclusion, we have proposed a method of determining the switching rates of a random telegraph noise, which is based on an analytical expression for the distribution of the filtered signal, and which should work even if the detector is slow. We have confirmed the validity of the model applying it to the telegraph signal generated by a single-electron transistor, for which the switching rates can be determined by a conventional threshold algorithm. Subsequently, we have demonstrated the efficiency of the method applying it to the device with a slow detector, for which the current threshold between the two states cannot be defined. We believe that

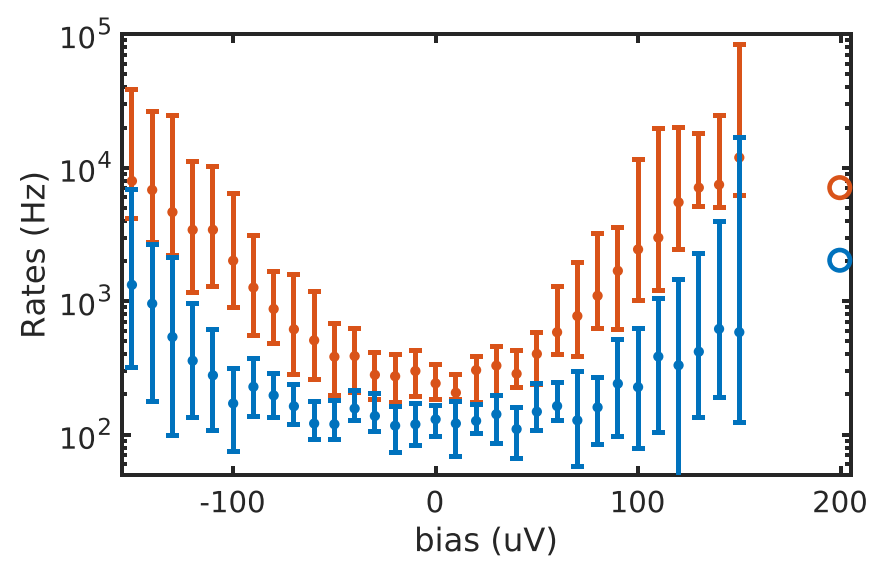

FIG. 6. Switching rates as a function of source SET bias voltage for sample B. Bias dependence of the tunneling rates $\gamma_{\uparrow}$ (red circles) and $\gamma_{\downarrow}$ (blue circles) for sample B for a fixed value of the gate voltage, $C_{g} V_{g} / e=0.42$, applied to the SET. The rates are extracted with traditional threshold-based methods, with error bars originating from measurement limitations, like small drift in gate voltage between different bias voltages and error in choice of effective detector bandwidth while using the approach of Ref. 10 for rate calculation. Open circles are the rates calculated with the method presented here. 
the formalism presented here can be extended to multi-level telegraph noise.

We acknowledge helpful discussions with Ivan Khaymovich. We acknowledge the provision of facilities by Aalto University at OtaNano Micronova Nanofabrication Centre. We thank Matthias Meschke for technical assistance. This work was partially supported by Academy of Finland, Project Nos. 284594, 272218, and 275167 (S.S., D.S.G., J.T.P., and J.P.P.).

${ }^{1}$ M. B. Weissman, Rev. Mod. Phys. 60, 537 (1988).

${ }^{2}$ M. J. Kirton and M. Uren, Adv. Phys. 38, 367 (1989).

${ }^{3}$ Z. Li, M. Sotto, F. Liu, M. K. Husain, H. Yoshimoto, Y. Sasago, D. Hisamoto, I. Tomita, Y. Tsuchiya, and S. Saito, Sci. Rep. 8, 250 (2018).

${ }^{4}$ O. Naaman and J. Aumentado, Phys. Rev. B 73, 172504 (2006).

${ }^{5}$ T. M. Buehler, D. J. Reilly, R. P. Starrett, V. C. Chan, A. R. Hamilton, A. S. Dzurak, and R. G. Clark, J. Appl. Phys. 96, 6827 (2004).

${ }^{6}$ M. Hofheinz, X. Jehl, M. Sanquer, G. Molas, M. Vinet, and S. Deleonibus, Eur. Phys. J. B 54, 299 (2006).

${ }^{7}$ M. Möttönen, R. de Sousa, J. Zhang, and K. B. Whaley, Phys. Rev. A 73, 022332 (2006).

${ }^{8}$ M. Shaw, R. Lutchyn, P. Delsing, and P. Echternach, Phys. Rev. B 78, 024503 (2008).

${ }^{9}$ G. J. Grabovskij, T. Peichl, J. Lisenfeld, G. Weiss, and A. V. Ustinov, Science 338, 232 (2012).

${ }^{10}$ O. Naaman and J. Aumentado, Phys. Rev. Lett. 96, 100201 (2006).
${ }^{11}$ Y. Yuzhelevski, M. Yuzhelevski, and G. Jung, Rev. Sci. Instrum. 71, 1681 (2000).

${ }^{12}$ J. Martin-Martinez, J. Diaz, R. Rodriguez, M. Nafria, and X. Aymerich, IEEE Electron Device Lett. 35, 479 (2014).

${ }^{13}$ B. Küng, O. Pfäffli, S. Gustavsson, T. Ihn, K. Ensslin, M. Reinwald, and W. Wegscheider, Phys. Rev. B 79, 035314 (2009).

${ }^{14}$ J. R. Prance, B. J. Van Bael, C. B. Simmons, D. E. Savage, M. G. Lagally, M. Friesen, S. N. Coppersmith, and M. A. Eriksson, Nanotechnology 26, 215201 (2015).

${ }^{15}$ S. Gustavsson, R. Leturcq, M. Studer, I. Shorubalko, T. Ihn, K. Ensslin, D. C. Driscoll, and A. C. Gossard, Surf. Sci. Rep. 64, 191 (2009).

${ }^{16}$ N. Ubbelohde, C. Fricke, C. Flindt, F. Hohls, and R. J. Haug, Nat. Commun. 3, 612 (2012).

${ }^{17}$ D. H. Slichter, R. Vijay, S. J. Weber, S. Boutin, M. Boissonneault, J. M. Gambetta, A. Blais, and I. Siddiqi, Phys. Rev. Lett. 109, 153601 (2012).

${ }^{18}$ L. R. Rabiner, Proc. IEEE 77, 257 (1989).

${ }^{19} \mathrm{H}$. Awano, H. Tsutsui, H. Ochi, and T. Sato, in 14th International Symposium on Quality Electronic Design (ISOED) (IEEE, 2013).

${ }^{20}$ F. M. Puglisi and P. Pavan, ECTI Trans. Electr. Eng., Electron., Commun. 12, 24 (2014); available at https://ecti-eec.org/index.php/ecti-eec/article/ view/135.

${ }^{21}$ G. Giusi, F. Crupi, and C. Pace, Rev. Sci. Instrum. 79, 024701 (2008).

${ }^{22}$ R. Fitzhugh, Math. Biosci. 64, 75 (1983).

${ }^{23}$ N. J. Lambert, A. A. Esmail, F. A. Pollock, M. Edwards, B. W. Lovett, and A. J. Ferguson, Phys. Rev. B 95, 235413 (2017).

${ }^{24}$ H. P. Breuer and F. Petruccione, The Theory of Open Quantum Systems (Oxford University Press, 2007).

${ }^{25}$ S. Singh, J. T. Peltonen, I. M. Khaymovich, J. V. Koski, C. Flindt, and J. P. Pekola, Phys. Rev. B 94, 241407(R) (2016).

${ }^{26}$ A. N. Jordan and E. V. Sukhorukov, Phys. Rev. Lett. 93, 260604 (2004). 